nephron

Practice
Nephron 2019;142:83-90

DOI: $10.1159 / 000497385$
Received: November 16, 2018

Accepted: January 30, 2019

Published online: March 21, 2019

\title{
Predicting Risk of Recurrent Acute Kidney Injury: A Systematic Review
}

\author{
Hilda O. Hounkpatin ${ }^{a}$ Simon D.S. Fraser ${ }^{b}$ Liz Glidewell ${ }^{c} d \quad$ Thomas Blakeman ${ }^{e}$ \\ Andrew Lewington ${ }^{f} g$ Paul J. Roderick ${ }^{b}$

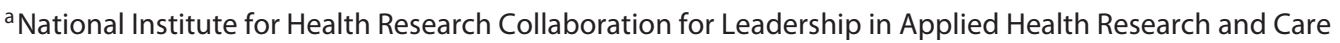 \\ (NIHR CLAHRC) Wessex Primary Care and Population Sciences, Faculty of Medicine, University of Southampton, \\ Southampton, UK; ${ }^{\mathrm{b}}$ Academic Unit of Primary Care and Population Sciences, Faculty of Medicine, University of \\ Southampton, Southampton, UK; ${ }^{C}$ Department of Health Sciences, University of York, York, UK; ${ }^{d}$ Leeds Institute of

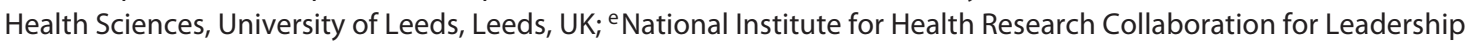 \\ in Applied Health Research and Care (NIHR CLAHRC) Greater Manchester, Centre for Primary Care, Institute of \\ Population Health, The University of Manchester, Manchester, UK; ${ }^{f}$ Department of Renal Medicine, Leeds Teaching \\ Hospitals NHS Trust, Leeds, UK; ${ }^{9}$ National Institute for Health Research (NIHR) Leeds In Vitro Diagnostic Cooperative, \\ Leeds, UK
}

\section{Keywords}

Acute renal injury $\cdot$ Recurrence $\cdot$ Epidemiology $\cdot$ Prognosis

\begin{abstract}
Background: Although the epidemiology of acute kidney injury (AKI) has been well described, less is known about recurrent $\mathrm{AKI}(\mathrm{r}-\mathrm{AKI})$. We undertook a systematic review to identify incidence, risk factors, and outcomes of $r$-AKI. Methods: MEDLINE, EMBASE, CINAHL, Cochrane, Web of Science were searched, from inception to December 2017, for quantitative studies on adults with AKI, where follow-up included reporting of $\mathrm{r}$-AKI. Two reviewers independently identified studies and assessed study quality. Summary: From 2,824 citations, 10 cohort studies met inclusion criteria (total patients $n=538,667$ ). There were 2 distinct set of studies; 4 studies assessed $\mathrm{r}$-AKI within the same hospital admission (most were intensive care unit [ICU] patients) and 6 studies as-
\end{abstract}

\section{KARGER}

(c) 2019 S. Karger AG, Basel

E-Mail karger@karger.com

www.karger.com/nef sessed postdischarge $r-A K I$. The median percentage of people developing $r$-AKI within the same hospital admission was 23.4\% (IQR 20.3-27.2\%) and postdischarge r-AKI was 31.3\% (IQR 26.4-33.7\%). A higher Acute Physiology and Chronic Health Evaluation score was associated with increased risk of r-AKI within the same hospital admission in ICU patients. Cardiovascular disease and AKI severity were associated with increased risk of postdischarge r-AKI. R-AKI (within same admission and postdischarge) was associated with worse survival. It was not possible to pool results due to methodological differences across studies, such as varying definitions for AKI and r-AKI, varying length of follow-up and effect measures. Key messages: More representative population-based studies with robust assessment of predictors and consensus definition of r-AKI are needed to identify risk factors and develop risk stratification tools to reduce recurrence and improve outcomes. Systematic Review Registration: CRD42017082668. 


\section{Introduction}

Acute kidney injury (AKI) is common and associated with poor clinical outcomes, including increased risk of development and progression of chronic kidney disease (CKD), and mortality [1-3]. The incidence of AKI has been well described, particularly for AKI acquired in hospital, but less is known about which factors contribute to adverse outcomes for people with AKI, particularly recurrent AKI (r-AKI). Recent studies have shown that many AKI survivors, including those with complete recovery, may experience further AKI episodes and that each $\mathrm{r}$-AKI episode further increases the risk of developing advanced CKD and mortality [4-6]. These findings suggest that strategies aimed at prevention of r-AKI may help improve long-term outcomes of AKI survivors. Preventing r-AKI is an important component of reducing AKI incidence and associated deaths, in line with the aims of the International Society of Nephrology 0by25 initiative [7].

Previous studies of $r$-AKI have reported varying rates and risk factors for r-AKI, likely due to the different populations and factors being studied, as well as differences in study sample size and length of follow-up. For example, some studies have reported no significant predictors for r-AKI [8-9], while other studies have indicated patient and clinical factors (such as older age, diabetes, volume depletion) predict $r$-AKI [5-6]. In order to improve understanding of incidence and predictors, this study reviews the current evidence on risk and outcomes of $r$ AKI.

\section{Materials and Methods}

A systematic review of quantitative studies was undertaken in accordance with Cochrane Systematic Reviews and the Centre for Reviews and Dissemination recommendations [10], and we have reported our findings according to PRISMA reporting guidelines $[11,12]$.

The study protocol was registered a priori with PROSPERO (PROSPERO 2017 CRD42017082668). Ethical approval was not required for this study as there was no patient-identifiable data.

\section{Search Strategy}

The databases Ovid MEDLINE, Ovid EMBASE, EBSCO CINAHL, Cochrane, and Web of Science were searched from inception to December 2017 using a combination of search terms for AKI (acute renal failure, acute kidney injury, acute renal insufficiency), recurrence (recurrence, repeat, re-occur), and prognosis (prognosis, predict, course). Search strategies for each database are available in online supplementary Appendix 1 (for all online suppl. material, see www.karger.com/doi/10.1159/000497385). Reference lists of relevant studies and review articles, conference pro- ceedings, websites of kidney organizations (such as ERA-EDTA, British Renal Society, Kidney Research UK, The Renal Association) were also searched. We also conducted citation-tracking and additional searches through Google ${ }^{\circledR}$. Searches were limited to studies on adult humans, published in the English language, and consisting of at least 100 study participants. Authors of identified studies were contacted and asked for clarification where necessary.

\section{Study Selection}

Two reviewers (H.O.H., S.D.S.F.) independently screened the titles and abstracts of all identified studies using a priori selection criteria. Both reviewers independently assessed full text of potentially relevant studies and determined eligibility. Discrepancies were resolved through discussion, though a third reviewer (P.J.R.) was available for adjudication if needed.

Quantitative studies were included if they described an adult population with AKI acquired in any setting (hospital or community) where follow-up included the reporting of r-AKI. We excluded studies with a small sample size (less than 100 people) in order to exclude case series, qualitative studies, and very small cohorts with potential for high risk of bias [13]. Studies conducted on children and case reports (consisting of less than 3 cases) were also excluded.

\section{Data Extraction and Risk of Bias Assessment}

One reviewer (H.O.H.) extracted information from the included studies into tables (developed a priori), with confirmation by a second reviewer (S.D.S.F.). Study characteristics extracted from eligible papers were author name, year of publication, study location, study aims, study design, study population details (sample size, population), outcomes, results, and conclusions.

Two reviewers independently assessed risk of bias using the Newcastle-Ottawa scale [14] since all included studies were observational studies rather than intervention studies. This scale has been designed for observational studies and is recommended by the Cochrane Collaboration $[10,14]$. The Newcastle-Ottawa scale uses a star system (with a maximum of 9 stars) to evaluate 3 domains: selection, comparability, and outcome bias. Selection bias refers to the representativeness of the exposed cohort, selection of the nonexposed cohort, ascertainment of exposure and outcome, demonstration that outcome of interest was not present at start of study. Comparability bias refers to adjustment for potential covariates (e.g., age, comorbidities), and outcome bias refers to assessment of outcome, length, and adequacy of follow-up. Each item was classified as at high, low, or unclear risk of bias, and overall level of bias for each study was achieved by adding the number of stars in each domain. Following the Newcastle-Ottawa scale methods, good quality was defined as scoring 3-4 stars in the selection domain, 1-2 stars in comparability domain, and 2-3 stars in the outcome domain. Fair quality was defined as 2 stars in the selection domain, 1-2 stars in the comparability domain, and 2-3 stars in outcome domain. Poor quality was defined as $0-1$ star in the selection domain, 0 stars in the comparability domain, and 0-1 star in the outcome domain.

\section{Data Synthesis and Statistical Analyses}

Studies were grouped according to whether they assessed r-AKI within the same hospital admission or recurrence postdischarge. Within each group, incidence across different clinical settings was reported. Heterogeneity across studies in each group was assessed 
Fig. 1. Flow diagram.

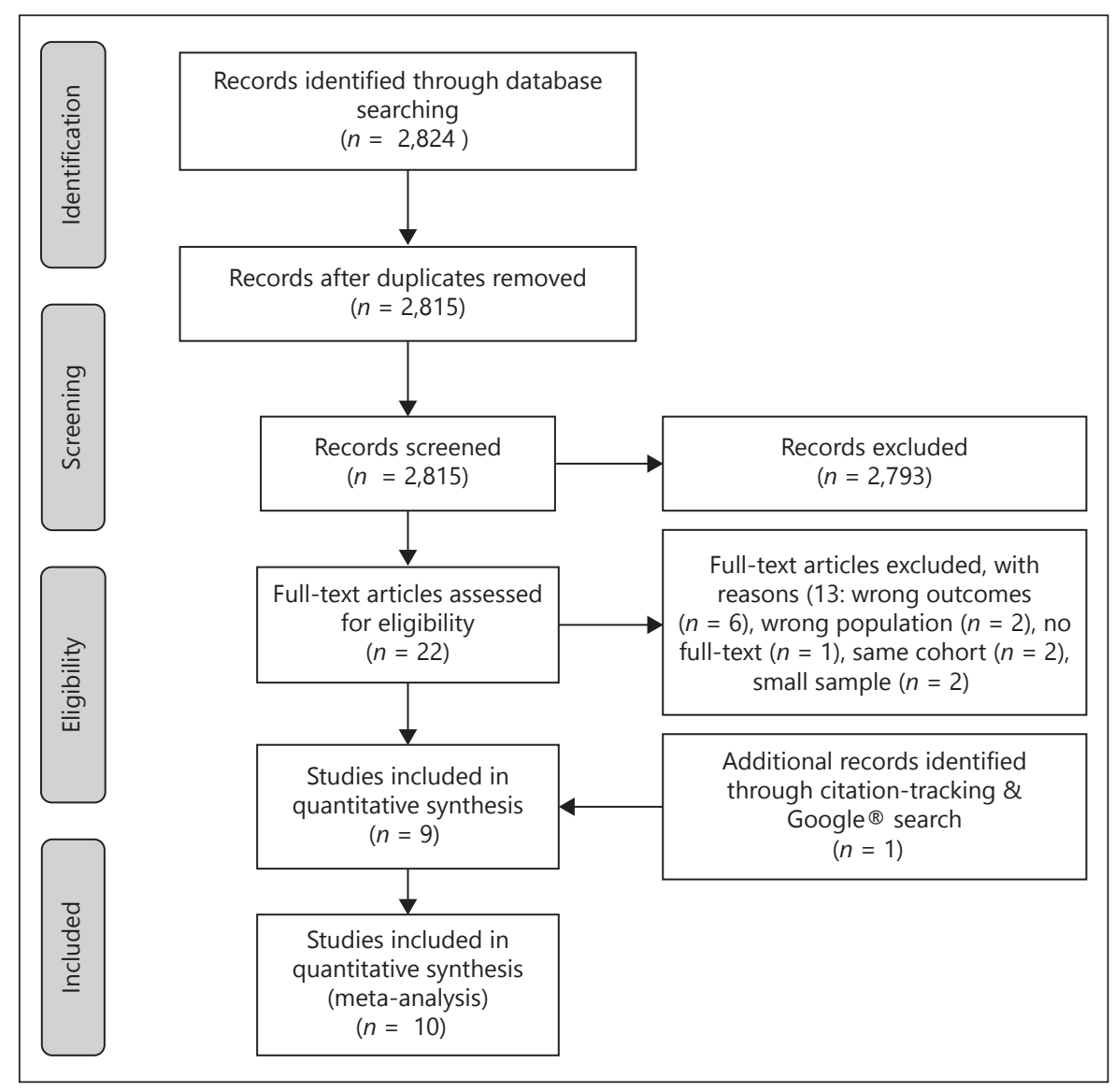

using the $I^{2}$ statistic (tested by Higgins $I^{2}$ test). Substantial statistical heterogeneity was considered to be present if the $I^{2}$ statistic was $>50 \%$ [10]. A narrative synthesis approach was taken for studies that were considered too clinically heterogeneous to pool. Where data were considered statistically and clinically homogenous, a pooled proportion (and 95\% CI) of the incidence of $\mathrm{r}$-AKI in each clinical group was estimated. Forest plots displaying incidence with the corresponding $95 \%$ CIs for each study were generated. A weighted random-effects model was used to pool incidence rates.

Risk factors from included studies in each group were summarized descriptively. Meta-analyses of pooled ORs from studies that provided adjusted ORs for potential risk factors were considered if studies proved to be sufficiently homogeneous. Outcomes from included studies in each group were summarized descriptively.

\section{Results}

Figure 1 presents a flow chart of the systematic review process. The searches identified 2,824 references. After screening titles and abstracts, 22 studies were selected for full-text review. Of these studies, 10 were excluded as they did not meet inclusion criteria. Of the 12 eligible studies,
1 full paper and 2 abstracts were based on the same study population, and full text was not available for 1 study, resulting in 9 unique studies. One conference abstract and 1 additional paper (based on the same study cohort) were identified through citation tracking and Google ${ }^{\circledR}$ searches. The final inclusion consisted of 10 unique studies ( $n=538,667$ patients).

Online supplementary Table S1 presents the characteristics of each included study. Studies were published between 2004 and 2018. Six studies were in a US population, 2 in a Spanish population, and 1 each in Canada and the United Kingdom. Each was a cohort study (7 retrospective and 3 prospective). Six studies examined general hospitalized (acute, emergency, elective admissions) patients (including 1 that focused on diabetic patients and 1 that focused on patients with severe sepsis), 3 studies examined general intensive care unit (ICU) patients, and 1 study focused on patients undergoing percutaneous coronary interventions (PCI: including elective, emergent, urgent, or salvage). Sample size of studies ranged from 170 to 453,475 (median 2,353; IQR 370.5-15,646.8) patients. Ascertain- 


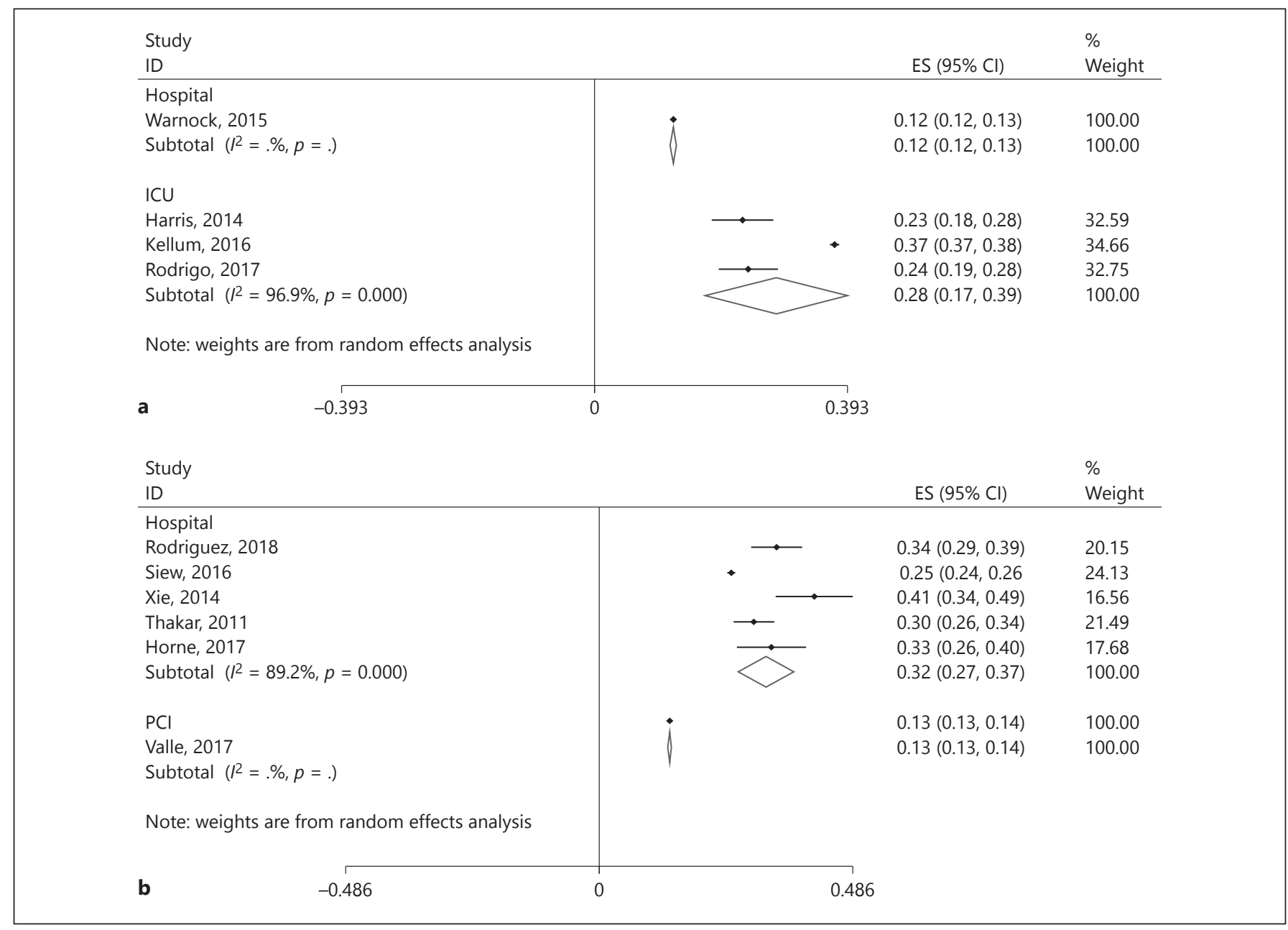

Fig. 2. a Random effects model showing proportion of people with $\mathrm{r}$-AKI within the same hospital admission. $\mathbf{b}$ Random effects model showing proportion of people with postdischarge r-AKI. ICU, intensive care unit; PCI, percutaneous coronary interventions.

ment of AKI and r-AKI was obtained through medical records [15-16], health-care databases $[4,5,9,17]$, regional administration database $[6,18]$, or national data registries [19]. Studies used varying definitions of AKI, based on serum creatinine: RIFLE $[6,9,16]$, $\operatorname{KDIGO}[5,15]$, or AKIN criteria $[4,18-20]$. There were 2 distinct set of studies; some studies assessed recurrence within the same hospital admission only $(n=4)$ and some studies assessed postdischarge recurrence up to 10 years $(n=6)$.

\section{Quality Assessment}

Based on the Newcastle-Ottawa scale, 6 of the 10 studies were rated overall good quality (6-8 stars) [4-6, 15, 18-19], 3 studies were deemed fair quality (5-6 stars) $[16,17,20]$, and 1 study was rated poor quality $(0-5$ stars, with 0 stars in the comparability domain) [9]. Online supplementary
Table S2 presents quality assessment domains for each study. Studies rated fair or good quality accounted for $99.6 \%(82,186 / 82,482)$ of the total AKI population across studies. Length of follow-up varied across studies (median 227.5 days; IQR 74.5-512.8 days for studies on r-AKI within same admission; median 3.5 years; IQR $1.5-4$ years for studies on $\mathrm{r}$-AKI postdischarge). Most of the studies rated good quality assessed independent effect of factors such as sex, age, and comorbidities (e.g., diabetes mellitus, cardiovascular disease) and AKI severity. Most studies did not include any statement on loss to follow-up.

\section{Incidence, Predictors, and Consequences of $r$-AKI}

The total population of people with AKI in studies assessing $\mathrm{r}$-AKI within the same admission was 35,943, of whom 8,712 (24\%) had r-AKI. There was considerable het-
86

Nephron 2019;142:83-90 DOI: $10.1159 / 000497385$
Hounkpatin/Fraser/Glidewell/Blakeman/ Lewington/Roderick 
erogeneity across studies $\left(I^{2}=96.9 \%, p<0.01\right)$. Figure $2 \mathrm{a}$ shows the percentage of people with $\mathrm{r}$-AKI within the same hospital admission. Three out of 4 studies assessing $r$-AKI within the same hospital admission were in an ICU population. The remaining study focused on transient hospitalacquired AKI patients who recovered and then subsequently developed hospital-acquired AKI. The median percentage of people developing $\mathrm{r}$-AKI within the same admission was $23.4 \%$ (IQR: 20.3\%, 27.2\%).

The total population of people with AKI in studies assessing postdischarge AKI was 52,786, of whom 8,732 (17\%) had r-AKI. There was considerable heterogeneity across studies $\left(I^{2}=89.2 \%, p<0.01\right)$. Figure $2 \mathrm{~b}$ shows the percentage of people with $\mathrm{r}$-AKI postdischarge. The median percentage of people developing r-AKI postdischarge (median follow-up: 3.5 years) was 31.3\% (IQR: $26.4 \%, 33.7 \%)$. Incidence of $\mathrm{r}$-AKI postdischarge was lowest for patients undergoing PCI (13\%, follow-up: 1 year) and highest for general hospitalized patients (median: $33.0 \%$, IQR: $29.6 \%, 34.0 \%$, median follow-up: 3 years).

Risk factors for $\mathrm{r}$-AKI varied across studies. Online supplementary Table S3 presents risk factors evaluated in at least 2 studies. A table showing the full list of risk factors assessed in all of the studies is available in the online supplementary Appendix (online suppl. Table S4). Most studies used multivariable models to evaluate independent effects of each factor. Studies on r-AKI within the same admission that assessed common factors reported that a higher Acute Physiology and Chronic Health Evaluation score (indicating greater severity of disease) was associated with increased risk of $r$-AKI. There were mixed findings on association of age, AKI severity, and diabetes with $\mathrm{r}$-AKI for this group. One study each identified that black race, lower baseline eGFR, and higher creatinine were associated with increased risk of $r$-AKI within the same hospital admission.

Studies on postdischarge $\mathrm{r}$-AKI reported that cardiac disease and AKI severity were associated with recurrence postdischarge. Siew et al. [6] divided cardiac disease into coronary artery disease, congestive heart failure, and acute coronary syndrome. All were significantly associated with $\mathrm{r}$-AKI, though hazard ratios varied. There were mixed findings for diabetes as a risk factor for $\mathrm{r}$-AKI across studies that assessed $\mathrm{r}$-AKI postdischarge. One study each found that lower baseline eGFR, higher creatinine, increasing albuminuria, advanced liver disease, malignancy, volume depletion (independent of degree of index AKI severity), nephrology follow-up care, and post PCI care were associated with postdischarge r-AKI.
Of the studies on $\mathrm{r}$-AKI within the same admission, 2 studies assessed short-term mortality (28 and 90 days) and 2 assessed 1-year mortality. R-AKI within the same hospital admission was associated with longer inpatient stay and worse short- (28 day) and longer-term survival $[5,9,15,19]$. R-AKI postdischarge was associated with higher risk of development of CKD, CKD progression, cardiovascular events, and long-term mortality $[4,6,17$, 20].

\section{Quantitative Data Synthesis}

It was not possible to pool results on risk factors due to heterogeneity across studies. Studies used different populations and evaluated different risk factors. Few studies reported effect estimates and those that did used different effect measures (ORs vs. hazard ratios). Length of follow-up also varied across the studies assessing postdischarge r-AKI.

\section{Discussion}

This systematic review identified that $\mathrm{r}$-AKI, both within the same hospital admission (largely on highrisk ICU patients) and postdischarge, was common. It is likely that the overall population incidence of $\mathrm{r}$-AKI is higher than observed here, as these studies focused on patients admitted to hospital and did not capture people with $\mathrm{r}$-AKI in the community who are not admitted to hospital. A higher Acute Physiology and Chronic Health Evaluation score was associated with increased risk of recurrence within the same hospital admission, and AKI severity and cardiovascular disease were associated with increased risk of recurrence postdischarge. Only 1 study considered heart failure as a separate entity [6]. Smaller studies reported no significant predictors for AKI recurrence, likely due to insufficient statistical power. Both r-AKI within an admission and postdischarge were associated with poorer outcomes.

There was incomplete evidence on risk factors for recurrence as individual studies were based on different populations and assessed different risk factors. In addition to that, studies had varying definitions of $r$-AKI, both in terms of context (r-AKI within same hospital admission versus postdischarge) and how recurrence was measured (index creatinine compared to most recent creatinine post recovery from prior AKI episode versus admission creatinine at $\mathrm{r}$-AKI hospital admission versus twofold increase in creatinine within $48 \mathrm{~h}$ ). The incon- 
sistency in how r-AKI was measured may have resulted in biased estimates of rates of recurrence as well as biased associations of risk factors with $\mathrm{r}$-AKI. An international consensus on the definition of $\mathrm{r}$-AKI is needed to allow more accurate estimates to be achieved and allow direct comparison across studies. Some studies that assessed rAKI within the same hospital admission had a short follow-up period that may not have been sufficient to observe $\mathrm{r}$-AKI, and studies on $\mathrm{r}$-AKI postdischarge had varying lengths of follow-up. More studies that assess postdischarge r-AKI (with similar length of follow-up) and examine associations of $\mathrm{r}$-AKI with quality of care and health system factors are needed to better understand these findings and how and to what extent risk of r-AKI may be prevented. Only 2 of the postdischarge $r$ AKI studies considered the severity of index AKI. Future studies should assess AKI severity as AKI severity has been found to be a strong predictor of AKI outcomes [21]. Finally, no studies were identified that assessed r-AKI in a community setting - an area that needs exploring as a significant proportion of AKI in the population is not admitted community acquired [22]. It is likely the AKI in hospital studies includes both communityacquired and hospital-acquired AKI, but studies did not distinguish between the 2 . There is a need for population-based studies that include all new incident AKI, particularly community-acquired AKI, in order to understand potentially modifiable factors in the community such as infection, medication, and fluid management. Finally, more work is needed to better understand the heterogeneous AKI population and characteristics (such as older age, more advanced CKD) of patient groups who develop r-AKI causing hospital admission and worse prognosis.

\section{Strength and Limitations}

The meta-analysis pooled data from 86,180 AKI patients to estimate proportions of people with $\mathrm{r}$-AKI. Most of the data were from large population-based or multicenter studies, therefore allowing results to be generalizable and less prone to selection bias. Statistical heterogeneity between studies was high, which may be expected as studies differed in their inclusion criteria, duration of follow-up, ascertainment method, definition of $\mathrm{r}$-AKI.

A limitation was the varying definitions of AKI and $r$ AKI (online suppl. Table S1a, b), which made it difficult to pool findings across all studies. Terminology for recurrence also varied across studies. Furthermore, 4 of the 10 studies did not describe patient attrition, and 1 study that reported high attrition did not report whether character- istics differed for those lost and not lost to follow-up. A key limitation was that many studies did not report effect measures of risk factors on $\mathrm{r}$-AKI and studies that did report used different effect measures. Some risk factors were assessed in only 1 study, which prevented inclusion into meta-analyses of risk factors for $\mathrm{r}$-AKI. Due to these reasons, as well as high heterogeneity across studies, it was not possible to perform meta-analysis of risk factors for $\mathrm{r}$-AKI. A further limitation was not all studies excluded dialysis (ESRD) patients, which may have resulted in over ascertainment of AKI, for example, due to spurious changes in creatinine levels. Similarly, AKI patients undergoing acute dialysis patients may also have been included in the studies, which may also lead to misclassification bias. This is due to an increase in creatinine levels after discontinuing dialysis for AKI, which may be misclassified as recurrent episode of AKI. Furthermore, some studies assessing $\mathrm{r}$-AKI postdischarge did not have access to outpatient data and only used inpatient creatinine values to assess $\mathrm{r}$-AKI. This may have resulted in under ascertainment of AKI episodes, particularly those occurring in the community. Additional limitations included lack of detail on time course of recurrence and the short follow-up period, particularly for studies assessing $\mathrm{r}$-AKI within same admission. It is likely people who don't recover during the index admission may eventually recover and experience $\mathrm{r}$-AKI. Individual studies may have been limited by the data captured in electronic health records and other clinically important variables may have been omitted. We used a recurrence search term to narrow the field, as the outcome of interest was recurrence. This means that any study that examined recurrence but did not include "recurrence" as a key word will not have been captured in our searches. Finally, most studies were on US populations and there were a lack of studies in other areas.

Implications for Clinical Practice and Future Research

The finding that $\mathrm{r}$-AKI is common supports recent recommendations that patients should be followed up within 3 months of having an AKI episode and monitored for renal and nonrenal events [23]. High-risk patients (e.g., older patients, patients with cardiovascular disease, patients with more severe AKI) may be followed up more closely and may benefit from individualized care based on patient characteristics, characteristics of the AKI episode, and degree of renal recovery [23]. The finding that AKI severity and cardiovascular disease (an adverse outcome of initial AKI) increase the risk of $r$ AKI highlight the importance of AKI prevention strate-
88

Nephron 2019;142:83-90 DOI: $10.1159 / 000497385$
Hounkpatin/Fraser/Glidewell/Blakeman/ Lewington/Roderick 
gies. There is a need for more research (using robust assessment of predictors and consensus definition of $\mathrm{r}$ AKI) to identify risk factors post initial AKI in those who survive to discharge and with long-term follow-up in order to develop risk stratification tools to improve post AKI care, prevent recurrence, and improve outcomes. Such studies may also lead to development and validation of prediction scores for poor outcomes, while recognizing the heterogeneity of the AKI population. Use of routine data may reduce the problem of loss to follow-up and allow better tracking of recurrence and other outcomes.

\section{Acknowledgment}

The authors would like to thank Brian Yuen, Primary Care and Population Sciences, University of Southampton for his advice on statistical methods.

\section{Ethics Statement}

The authors have no ethical conflicts to disclose.

\section{Disclosure Statement}

The authors have no conflicts of interest to declare.

\section{Funding Sources}

H.O.H. is funded by the National Institute for Health Research Collaboration for Leadership in Applied Health Research and Care (NIHR CLAHRC) Wessex at Southampton NHS Hospitals Foundation Trust. T.B. is funded by the NIHR CLAHRC Greater Manchester. The funder had no role in the design of the study, data collection and analysis, decision to publish, or preparation of the manuscript. However, the project outlined in this document may be considered to be affiliated to the work of the NIHR CLAHRC Greater Manchester and Wessex. The views expressed in this document are those of the author(s) and not necessarily those of the NHS, NIHR or the Department of Health and Social Care.

\section{Author Contributions}

All authors conceived and refined the idea. $\mathrm{HH}$ conducted the literature searches, statistical analyses, and drafted the paper. P.J.R., S.D.S.F., A.L., T.B., and L.G. provided expert advice and support throughout. All authors reviewed and revised the manuscript and approved the final version.

\section{References}

1 Coca SG, Yusuf B, Shlipak MG, Garg AX, Parikh CR. Long-term risk of mortality and other adverse outcomes after acute kidney injury: a systematic review and meta-analysis. Am J Kidney Dis. 2009 Jun;53(6):961-73.

2 Coca SG, Singanamala S, Parikh CR. Chronic kidney disease after acute kidney injury: a systematic review and meta-analysis. Kidney Int. 2012 Mar;81(5):442-8.

3 Sawhney S, Mitchell M, Marks A, Fluck N, Black C. Long-term prognosis after acute kidney injury (AKI): what is the role of baseline kidney function and recovery? A systematic review. BMJ Open. 2015 Jan;5(1):e006497.

4 Thakar CV, Christianson A, Himmelfarb J, Leonard AC. Acute kidney injury episodes and chronic kidney disease risk in diabetes mellitus. Clin J Am Soc Nephrol. 2011 Nov; 6(11):2567-72.

5 Kellum JA, Sileanu FE, Bihorac A, Hoste EA, Chawla LS. Recovery after acute kidney injury. Am J Respir Crit Care Med. 2016;65:528-9.

6 Siew ED, Parr SK, Abdel-Kader K, Eden SK, Peterson JF, Bansal N, et al. Predictors of Recurrent AKI. J Am Soc Nephrol. 2016 Apr; 27(4):1190-200.

7 Acute Kidney Injury (AKI) is preventable and treatable. International Society of $\mathrm{Ne}$ phrology website. https://www.theisn.org/allarticles/616-0by25. Accessed April 16, 2018.
8 Bardak S, Turgutalp K, Türkegün M, Demir $S$, Kiykım A. Recurrent acute kidney injury in renal transplant patients: a single-center study. Transplant Proc. 2015 Jun;47(5):143741.

9 Harris DG, Koo G, McCrone MP, Scalea TM, Chiu WC, Diaz JJ, et al. Recurrent kidney injury in critically ill surgical patients is common and associated with worse outcomes. J Trauma Acute Care Surg. 2014 Jun;76(6): 1397-401.

10 Higgins JP, Green S. Cochrane Handbook for Systematic Reviews of Interventions Version 5.1.0 [updated March 2011]. The Cochrane Collaboration, 2011. Centre for Reviews and Dissemination. Systematic reviews: CRD's guidance for undertaking a systematic review York: CRD. University of York, 2009.

11 Centre for Reviews and Dissemination. Systematic reviews: CRD's guidance for undertaking a systematic review York: CRD. University of York, 2009.

12 Stewart LA, Clarke M, Rovers M, Riley RD, Simmonds M, Stewart G, et al.; PRISMA-IPD Development Group. Preferred Reporting Items for Systematic Review and Meta-Analyses of individual participant data: the PRISMA-IPD Statement. JAMA. 2015 Apr; 313(16):1657-65.
13 Turner RM, Bird SM, Higgins JP. The impact of study size on meta-analyses: examination of underpowered studies in Cochrane reviews. PLoS One. 2013;8(3):e59202.

14 Wells G, Shea B, O'Connell D, Peterson J, Welch V, Losos M, et al. The Newcastle-Ottawa Scale (NOS) for assessing the quality of nonrandomised studies in meta-analyses. 2013. http://www.ohri.ca/programs/clinical_ epidemiology/oxford.asp

15 Rodrigo E, Suberviola B, Santibáñez M, Belmar L, Castellanos Á, Heras M, et al. Association between recurrence of acute kidney injury and mortality in intensive care unit patients with severe sepsis. J Intensive Care. 2017 May;5(1):28.

16 Xie M, Iqbal S. Predictors for nephrology outpatient care and recurrence of acute kidney injury (AKI) after an in-hospital AKI episode. Hemodial Int. 2014 Oct;18 Suppl 1:S7-12.

17 Rodríguez E, Arias-Cabrales C, Bermejo S, Sierra A, Burballa C, Soler MJ, et al. Impact of recurrent acute kidney injury on patient outcomes. Kidney Blood Press Res. 2018;43(1): 34-44.

18 Warnock DG, Powell TC, Donnelly JP, Wang HE. Categories of hospital-associated acute kidney injury: time course of changes in serum creatinine values. Nephron. 2015;131(4): 227-36. 
19 Valle JA, McCoy LA, Maddox TM, Rumsfeld JS, Ho PM, Casserly IP, et al. Longitudinal risk of adverse events in patients with acute kidney injury after percutaneous coronary intervention: Insights from the national cardiovascular data registry. Circ Cardiovasc Interv. 2017;10(4):pii: e004439.

20 Horne KL, Packington R, Monaghan J, Reilly T, Selby NM. Three-year outcomes after acute kindey injury: results of a prospective parallel group cohort study. BMJ Open. 2017;7: e015316.

21 Hoste EA, Bagshaw SM, Bellomo R, Cely CM, Colman R, Cruz DN, et al. Epidemiology of acute kidney injury in critically ill patients: the multinational AKI-EPI study. Intensive Care Med. 2015 Aug;41(8):1411-23.

22 Sawhney S, Fluck N, Fraser SD, Marks A, Prescott GJ, Roderick PJ, et al. KDIGO- based acute kidney injury criteria operate differently in hospitals and the communityfindings from a large population cohort. Nephrol Dial Transplant. 2016 Jun;31(6): 922-9.

23 Silver SA, Adu D, Agarwal S, Gupta KL, Lewington AJ, Pannu N, et al. Strategies to enhance rehabilitation after acute kidney injury in the developing world. Kidney Int Rep. 2017;2(4):579-93. 